\title{
Common HYPERTENSION/IHD Risk Factors and Adherence to Treatment among the Population of Veliky Novgorod (based on Materials of "RELIEF" Regional Study)
}

\author{
Zhbanova N*, Yuhno M, and Prosorova I \\ Department of Education and Science, Russia
}

*Corresponding author: : Zhbanova N, Department of Education and Science, The-Wise

Novgorod State University, Russia.

Received Date: December 17, 2018

Published Date: January 04, 2019

Keywords: Risk factors; Relief regional study; Compliances

\section{Objective}

To study the prevalence of the major cardiovascular risk factors among the population of Veliky Novgorod and provide reasons for poor adherence to treatment in patients.

\section{Material and Methods}

By analogy with the "Regular Treatment and Prevention as The Key to Improving CVD Situation in Russia - RELIEF” large-scale study on assessing the adequacy of the treatment of hypertension and coronary heart disease and adherence of patients to the doctor's recommendations, a one-time, continuous, clinical and epidemiological study was conducted among 39 first contact physicians in Veliky Novgorod hospitals and 212 patients with a verified diagnosis of hypertension and coronary heart disease. Analytical review of obtained results was conducted with the use of standard algorithms of variation statistics, including stepwise logistic regression models.

\section{Result}

The prevalence of cardiovascular risk factors among the population of a typical North-West region was studied from the standpoint of evidence-based medicine, and mathematical modeling. The study tested the method of estimating adherence to treatment using variation statistics and multivariate analysis based on nonparametric statistics. Cross-surveying method was used, followed by the network data analysis. Reasons for low adherence to treatment among the 39 first contact physicians and their 212 patients were studied. Analysis of the socio-economic factors as major cardiovascular risk factors in the region was performed. It was established that the following factors have the main influence on adherence to treatment: factors associated with physicians, patients, disease, recommended therapy and socio-economic factors.

\section{Conclusion}

According to the results of a sample survey, incidence of major cardiovascular risk factors is higher among the population of Novgorod region than in Northwestern Federal District and the Russian Federation. Thus, the prevalence of hypertension is $47 \%$ on average (men $43.2 \%$, women $-49.3 \%$ ), grade 1 hypertension is typical for $10.7 \%$ of respondents; grade 2 - for $22.7 \%$ and grade 3 - for $13.6 \%$; among women - grade 1 - for $11.6 \%$, grade 2 - for $24 \%$, grade 3 - for $13.7 \%$; among men, respectively $8.8 \%, 20.9 \%$ and $13.5 \%$. The prevalence of obesity was: 1 degree obesity among women - 14.6\%, 2 degree $-4.6 \%$, 3 degree - 1.8\%; among men, respectively $8.6 \%, 2.0 \%$ and $0.8 \%$.

Dyslipidemia rate - on average - was identified among $26.3 \%$ of the population, including women $-26.7 \%$, and male $-25.5 \%$. The frequency of a combination of hypertension and obesity according to Odds ratio criterion was from 2.5 among men (1 degree) to 10.44 (3 degree), among women, respectively - from 3.5 to 13.2. Diabetis rate $-5.06 \%$ (men $-7.02 \%$; women - 2.89\%); IGT rate $-7.2 \%$ (men $-6.22 \%$; women $-7.07 \%$ ) exertional angina (regardless of FC - 
$17.5 \%$ among men, $13.5 \%$ among women). Rest angina $-1.47 \%$ among women, $1.72 \%$ among men. MI: $-2.86 \%$ among women, $5.9 \%$ among men. Maximum MI rate is $11.1 \%$ among men (age range 60-69 years) and 6.5\% among women (80 years and older) Lack of circulation: $8.64 \%$ among men, $10.1 \%$. among women.

In the course of this study, insufficient measures for weight adjustment in patients with hypertension and coronary heart disease, were noted as one of the leading risk factors; high degree of MI dependence from BMI were identified regardless of gender Dyslipidemia is indicated in patients regardless of gender and diagnosis, and in men such values as thyroglobulin, HDL and total cholesterol have the decisive importance in the comparative analysis of patients with myocardial infarction and coronary heart disease. It is proved that the estimate of the lipid spectrum is possible only in terms of descriptive and multivariate nonparametric statistics. It was found that the outcome of the disease is directly affected by psychological and emotional factors, dyslipidemia and obesity.
These study findings indicate a high level of complications among the respondents, as well as low level of compliance and knowledge about diseases, both at medical care and patients level. Twenty five- thirty percent of female respondents had cardiological complaints, mend had chest pains and headaches complaints. Frequency of compliance with medical recommendations among women is in the range of $57 \%$ (age group of 80 years and older), for males the range is $30-50 \%$ (mainly older age groups). According to respondents, side effects are the main cause of violations $(65.3 \%$ - in 70-79 age group), as well as drugs low effectiveness and their high cost. From 3 to $4.6 \%$ of the respondents are not aware of Hypertension / CHD disease. From few to none respondents are aware about the complications from diabetes.

\section{Acknowledgement}

None.

\section{Conflict of Interest}

No conflict of interest. 